\section{Determinantes do trabalho remunerado entre brasileiros mais velhos usando modelagem de equações estruturais: evidências do ELSI-Brasil}

\section{Determinants of paid work among older \\ Brazilians using structural equation modeling: evidence from the ELSI-Brazil study}

\section{Determinantes del trabajo remunerado entre adultos mayores brasileños usando modelos de ecuaciones estructurales: evidencias del ELSI-Brasil}

Camila Menezes Sabino de Castro 1 Maria Fernanda Lima-Costa 1,2 Jorge Alexandre Barbosa Neves 2 Fabiola Bof de Andrade 1 Rosana Ferreira Sampaio 2

\section{Resumo}

O objetivo do estudo foi desenvolver um modelo conceitual e explorar associações diretas $e$ indiretas entre trabalho remunerado e fatores que operam ao longo da vida em amostra nacional representativa da população brasileira com 50 anos e mais. A análise foi baseada em 8.903 participantes da linha de base do Estudo Longitudinal da Saúde dos Idosos Brasileiros (ELSI-Brasil). Os fatores de exposição foram sociodemográficos, de saúde, trabalho e interação social. Utilizando modelo de equações estruturais, observou-se que o trabalho remunerado apresentou associação total com a condição social ao longo da vida entre mulheres (coeficiente de padronização $-C P=0,489$ ) e associação direta com a capacidade para o trabalho entre homens $(C P=0,527)$. Apenas para as mulheres, associação indireta e negativa foi observada com o esforço físico intenso no trabalho ao longo da vida, via aposentadoria $(C P=$ -0,156). Homens com trabalho remunerado foram mais propensos à participação social, aferida pelo pertencimento a grupos ou associações $(C P=0,209)$. Entre as mulheres, essa participação ocorreu por meio de interações com família e amigos $(C P=0,047)$, via capacidade para o trabalho. Tanto entre homens quanto entre mulheres, a condição de saúde ao longo da vida mostrou associação indireta e positiva $(C P=0,298$ homens; $C P=0,142$ mulheres $)$ com o trabalho remunerado, via capacidade para o trabalho. Todos os fatores mencionados apresentaram associação significativa com o trabalho remunerado. Os resultados do estudo mostraram que a participação de homens e mulheres mais velhos no mercado de trabalho ocorre por mecanismos diferentes, principalmente ao considerar os fatores relacionados ao trabalho e interação

Envelhecimento; Trabalho; Modelos Estatísticos; Situação Sociecônomica; Interação Social

\author{
Correspondência \\ C. M. S. Castro \\ Instituto René Rachou, Fundação Oswaldo Cruz, \\ Belo Horizonte, Brasil. \\ Av. Augusto de Lima 1715, Belo Horizonte, MG \\ 30190-002, Brasil. \\ camilamscastro@gmail.com \\ 1 Instituto René Rachou, Fundação Oswaldo Cruz, \\ Belo Horizonte, Brasil. \\ 2 Programa de Pós-graduação em Saúde Púbica, \\ Universidade Federal de Minas Gerais, Belo Horizonte, Brasil.
}




\section{Introdução}

O envelhecimento populacional traz desafios significativos para o mercado de trabalho. Como alternativa para minimizar esse desafio, diversos países têm considerado a inclusão das pessoas mais velhas nas atividades 1,2,3. Estudos sobre ocupação utilizam, frequentemente, idade de 45 ou 50 anos como critério base para o termo "trabalhadores mais velhos". Esse ponto de vista oferece melhores possibilidades de medidas preventivas na saúde dos trabalhadores 4 .

Em nível mundial, a proporção da participação de adultos mais velhos no mercado de trabalho, em relação ao gênero, é maior entre os homens 5,6. Observa-se que, após um período de declínio constante, a participação dos homens mais velhos na força de trabalho aumentou recentemente na maioria dos países de alta renda da Organização para a Cooperação e Desenvolvimento Econômico (OECD) 5. Concomitantemente, a participação das mulheres de todas as idades no mercado de trabalho vem aumentando tanto em países de alta quanto de média renda 5. No Brasil, verificou-se a predominância dos homens $(56,1 \%)$ entre as pessoas ocupadas, o que foi confirmado em todas as regiões brasileiras ${ }^{6}$. Em relação à análise por grupos de idade, o segmento dos trabalhadores mais velhos foi o que apresentou as maiores taxas de crescimento da ocupação. No terceiro trimestre de 2019, comparado com o mesmo período de 2017 e 2018, observou-se uma expansão da participação dos adultos mais velhos no mercado de trabalho. A participação das pessoas de 40-59 anos de idade passou de 39,9\% para 40,4\%, e a participação das pessoas com 60 anos e mais passou de 7,6\% para $8,3 \%$. Por outro lado, houve uma diminuição da participação no mercado de trabalho das pessoas entre 18-39 anos 6.

A participação de adultos mais velhos no mercado de trabalho é um processo complexo, influenciado por experiências passadas e atuais da vida. Pesquisas em países de alta renda têm mostrado que a participação das pessoas mais velhas na força de trabalho não é determinada por um único fator. Características individuais (sexo, idade, status socioeconômico) e da família interagem com os fatores relacionados à organização do trabalho e com os fatores contextuais, como mercado de trabalho e cobertura do sistema previdenciário 2,7. Outro exemplo de complexidade na participação dos mais velhos na força de trabalho é que muitos determinantes desta participação estão sujeitos a mudanças substanciais e, às vezes, temporalmente muito rápidas 7,8 . Somando-se a isso, alguns estudos também mostraram que fatores biológicos, sociais e ambientais nas diferentes fases da vida podem acumular-se ao longo do tempo, impactando na tomada de decisão de permanecer trabalhando nas idades avançadas 9,10,11.

Existem poucos estudos brasileiros examinando os determinantes da participação de adultos mais velhos no mercado de trabalho. Pesquisa conduzida em São Paulo, no ano 2000, entre idosos (60 anos e mais), mostrou que a idade e a escolaridade diminuem seu poder explicativo para o trabalho remunerado nas idades mais velhas quando são consideradas as condições de saúde 12 . Um estudo mais recente, realizado na Região Metropolitana de Belo Horizonte, Minas Gerais, entre adultos mais velhos (50-69 anos de idade), mostrou que a associação entre autoavaliação de saúde e trabalho remunerado foi modificada pela escolaridade, principalmente entre as mulheres 13 . Em comparação àqueles com boa autoavaliação de saúde e escolaridade igual ou superior a oito anos (grupo de referência), a participação no trabalho remunerado foi duas vezes mais baixa entre os homens com pior autoavaliação de saúde, independentemente do nível de escolaridade. Para as mulheres com pior autoavaliação de saúde, a participação no trabalho remunerado foi cerca de nove vezes menor entre aquelas com escolaridade mais baixa 13 .

Frente a essas evidências, é preciso aprofundar na compreensão dos mecanismos pelos quais homens e mulheres em idades avançadas permanecem trabalhando. O modelo de equação estrutural tem sido cada vez mais utilizado na área da saúde para medir e testar relações em fenômenos complexos que exigem a análise de múltiplas relações simultaneamente 14,15. A vantagem da utilização do modelo de equação estrutural está no fato de que esta técnica estatística estima uma série de equações de regressão múltipla separadas, mas dependentes entre si, baseadas no modelo conceitual proposto pelo pesquisadores 16,17 . O presente estudo baseou-se em evidências da literatura nacional e internacional desenvolvidas em estudos longitudinais que investigaram especificamente os determinantes da participação na força de trabalho de pessoas em idades avançadas 2,8. Assim, o objetivo deste estudo foi 
desenvolver um modelo conceitual e explorar as associações diretas e indiretas entre fatores sociodemográficos, de saúde, trabalho, interação social e a participação no trabalho remunerado de homens e mulheres em amostra nacional representativa da população com 50 anos e mais.

\section{Métodos}

\section{Fonte de dados e amostra}

Foram usados dados do inquérito da linha de base do Estudo Longitudinal da Saúde dos Idosos Brasileiros (ELSI-Brasil), cuja amostra foi delineada para representar a população brasileira não institucionalizada, com idade igual ou superior a 50 anos. A coleta de dados da linha de base da coorte foi conduzida entre 2015 e 2016. A amostragem utilizou um delineamento complexo, combinando estratificação por municípios, setores censitários e domicílios. Todos os residentes nos domicílios selecionados com idade igual ou superior a 50 anos foram elegíveis para entrevista e outros procedimentos. A amostra final foi estimada em 10 mil pessoas (9.412 participaram), residentes em 70 municípios das cinco regiões do país. Mais detalhes estão disponíveis na homepage do ELSI-Brasil (http://elsi.cpqrr. fiocruz.br/) e em publicação anterior 18.

O ELSI-Brasil foi aprovado pelo Comitê de Ética em Pesquisa da Fundação Oswaldo Cruz, Minas Gerais (CAAE: 34649814.3.0000.5091). Todos os participantes assinaram o termo de consentimento livre e esclarecido separados para cada procedimento da pesquisa.

\section{Variáveis do estudo}

A variável dependente do estudo foi participação no trabalho remunerado obtida pela pergunta: "O(A) $\mathrm{Sr}$ (a). trabalhou remuneradamente nos últimos 30 dias?". Trabalho remunerado foi considerado como o exercício de qualquer atividade com remuneração em dinheiro, produtos, mercadorias ou benefícios. Essa variável incluiu assalariados, patrões, trabalhador autônomo, trabalho por produção e biscate, pessoas afastadas por motivo de férias, licença por doença, entre outros. Todas as outras condições, quais sejam aposentados, pedintes, estudantes, donas de casa que se dedicam exclusivamente aos afazeres domésticos, pessoas que trabalham sem remuneração ou se dedicam a trabalho voluntário e aqueles que viviam exclusivamente de renda ou ajuda de parentes e/ou conhecidos foram incluídos na categoria não trabalho.

As variáveis independentes foram definidas considerando-se os fatores de exposição que operam ao longo da vida e foram classificadas em fatores sociodemográficos e fatores relacionados à saúde, ao trabalho e à interação social. As variáveis sociodemográficas consideradas foram: (1) anos de escolaridade da mãe (0-4 anos vs. 5 anos ou mais); (2) local de residência aos 10 anos (rural vs. urbano); (3) idade em que começou a trabalhar (até 17 anos vs. 18 anos e mais); (4) anos de escolaridade do participante (0-7 anos vs. 8 anos ou mais); (5) idade na data da entrevista; (6) posição no domicílio (responsável pelo domicílio ou não); (7) situação conjugal (vive ou não com cônjuge ou companheiro(a); e (8) local de residência atual (rural ou urbano). Para a presente análise foi construída a variável latente condição social ao longo da vida, com base nas variáveis anos de escolaridade da mãe, local de residência aos 10 anos, idade em que começou a trabalhar e anos de escolaridade do participante. Essa variável foi baseada na análise de componentes principais e, posteriormente, foi dicotomizada em acima da média e abaixo deste valor.

As variáveis relacionadas à saúde foram: (1) saúde até os 15 anos de idade, aferida pela pergunta: "O(a) $\mathrm{Sr}(\mathrm{a})$. diria que a sua saúde, desde o nascimento até os 15 anos de idade, era:" boa (boa, muito boa ou excelente) vs ruim (razoável ou ruim); (2) autoavaliação da saúde atual, definida pela resposta à pergunta: “Em geral, como o(a) Sr(a). avalia a sua saúde?”, categorizada como boa (boa ou muito boa) vs. ruim (regular, ruim ou muito ruim); e (3) número de doenças crônicas, categorizado em pelo menos uma vs duas ou mais. As doenças foram definidas pela resposta à pergunta: "Algum médico já disse que o(a) Sr(a). tinha 'tal doença'?', sendo consideradas hipertensão, diabetes, colesterol, acidente vascular cerebral, asma, artrite ou reumatismo, osteoporose, problema crônico de coluna, câncer, doença do 
coração e depressão. Para a presente pesquisa, a variável latente condição de saúde ao longo da vida foi construída baseada na análise de componentes principais, com todas as variáveis descritas anteriormente. Em seguida, a variável foi dicotomizada em acima da média e abaixo deste valor.

As variáveis relacionadas ao trabalho foram: (1) esforço físico intenso no trabalho ao longo da vida, definido pela informação: "Como o(a) Sr(a). descreveria as exigências físicas do trabalho que exerceu durante a maior parte da vida?", categorizada como ausência de esforço físico intenso (nunca trabalhou ou ficava sentado a maior parte do tempo, ficava em pé/andando ou o trabalho exigia algum esforço físico) vs. presença de esforço físico intenso (o trabalho exigia esforço físico intenso a maior parte do tempo); (2) capacidade para o trabalho, definida pela pergunta: "Como o(a) Sr(a). avalia a sua capacidade atual para o trabalho?", categorizada como boa (boa/muito boa) vs. ruim (razoável/ruim/ muito ruim); e (3) aposentadoria ( $\mathrm{sim} /$ não), definido como aposentado quando o entrevistado declarou receber salário ou pensão do Instituto Nacional do Seguro Social (INSS) ou governo.

As variáveis relativas à interação social foram: (1) interação social primária, elaborada baseando-se na informação sobre a frequência com que a pessoa se encontrava ou conversava com filhos, parentes e/ou amigos, sem contar os que moravam com ele, categorizada como três ou mais vezes na semana, uma ou duas vezes por semana, uma ou duas vezes por mês, a cada dois ou três meses, uma ou duas vezes por ano e menos de uma vez por ano ou nunca; e (2) interação social secundária, elaborada com base na informação se o indivíduo tinha, nos últimos 12 meses, participado de atividades sociais organizadas ou associações civis, conselhos, lideranças comunitárias, cooperativas, partidos políticos etc, categorizada como não vs. sim. Para a interação social primária, as opções três ou mais vezes na semana e uma ou duas vezes por semana foram categorizadas em interação social elevada. Para a interação secundária, a categoria "sim" foi classificada em interação social elevada. No presente artigo usamos os termos "interação primária", que é uma proxy para o conceito de "laço forte", e "interação secundária", que é uma proxy para o conceito de "laço fraco" proposto por Granovetter 19.

\section{Modelo conceitual}

O modelo proposto foi desenvolvido tendo como base dois modelos teóricos da literatura internacional, o lidA Cohort Study (German Cohort Study on Work, Age, Health and Work Participation) 2 e o Study on Transitions in Employment, Ability and Motivation 8, com o intuito de explorar as relações diretas e indiretas entre os fatores associados à participação no trabalho remunerado de homens e mulheres brasileiros com 50 anos e mais. A condição social ao longo da vida ocupou a posição mais distal do modelo; as variáveis intermediárias foram condição de saúde ao longo da vida, esforço físico intenso no trabalho ao longo da vida, aposentadoria e interação social e, por último, a capacidade para o trabalho foi considerada a variável proximal na associação com o trabalho remunerado. A idade na data da entrevista foi usada como variável de ajuste para todas as associações do modelo. As variáveis posição no domicílio, situação conjugal e local de residência atual foram consideradas como variáveis de ajuste para o desfecho (trabalho remunerado). Na Figura 1, as formas circulares ou elípticas representam as variáveis latentes e os retângulos as variáveis observadas. As setas indicam as relações entre as variáveis, podendo ser diretas ou indiretas.

O modelo proposto assume que as variáveis condição social ao longo da vida, condição de saúde ao longo da vida, esforço físico intenso no trabalho ao longo da vida, capacidade para o trabalho, aposentadoria, interação social primária e interação social secundária estão associadas de forma direta com a participação no trabalho remunerado. Além disso, a condição social ao longo da vida está associada indiretamente ao trabalho remunerado, pelas vias da condição de saúde, trabalho e interação social.

\section{Análise dos dados}

$\mathrm{Na}$ análise descritiva dos participantes do estudo foi usado o teste qui-quadrado de Pearson, para examinar diferenças entre homens e mulheres. O modelo de equação estrutural foi usado para analisar, simultaneamente, os fatores associados ao trabalho remunerado. Esse método permite testar a validade de um modelo conceitual baseado em um conjunto de variáveis observáveis e latentes. Uma variável latente ou constructo é aquela que não pode ser observada diretamente, com base nas unidades de análise em estudo, mas supostamente está relacionada a muitas variáveis que podem ser 
Figura 1

Modelo conceitual desenvolvido para explorar as inter-relações entre as variáveis associadas à participação no trabalho remunerado de homens e mulheres com 50 anos ou mais. Estudo Longitudinal da Saúde dos Idosos Brasileiros (ELSI-Brasil), 2015-2016.

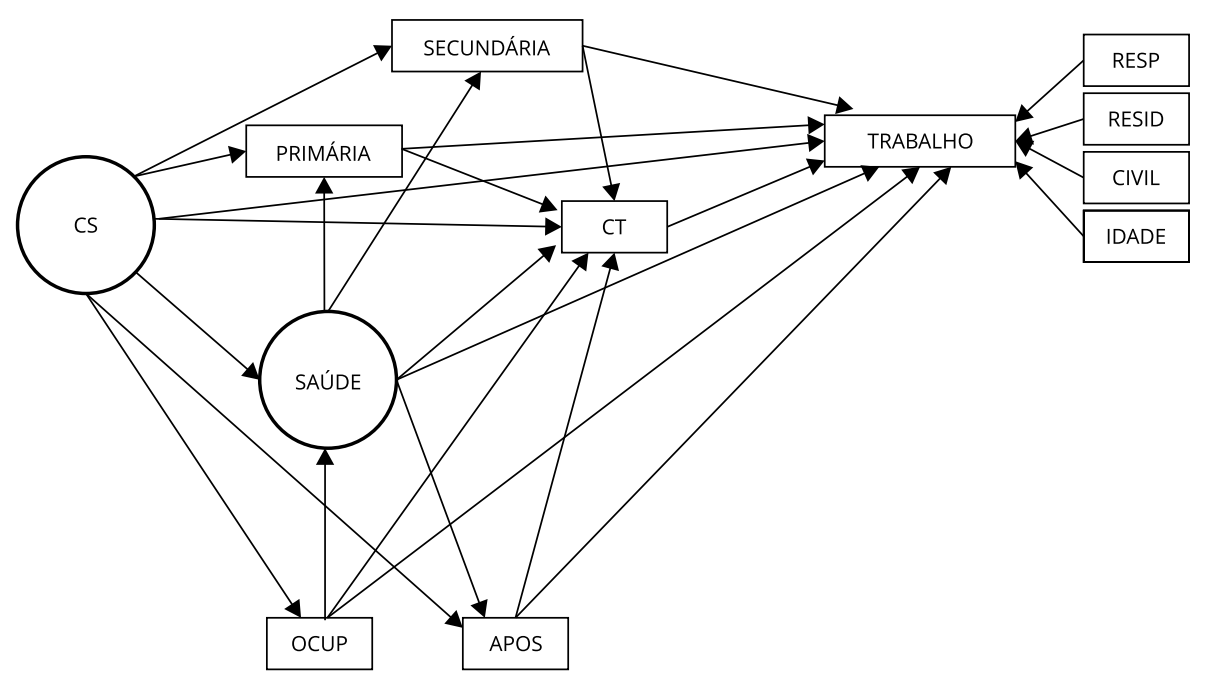

Fonte: elaboração própria dos autores (2018).

APOS: aposentadoria; CIVIL: situação conjugal; CS: condição social ao longo da vida (anos de escolaridade da mãe, local de residência aos 10 anos, idade em que começou a trabalhar, anos de escolaridade do participante);

CT: capacidade para o trabalho; IDADE: idade na data da entrevista; OCUP: esforço físico intenso no trabalho ao longo da vida; PRIMÁRIA: interação social primária; SAÚDE: condição de saúde ao longo da vida (saúde até os 15 anos de idade, autovaliação da saúde atual, número e doenças crônicas); SECUNDÁRIA: interação social secundária; RESP: posição no domicílio; RESID: local de residência atual; TRABALHO: trabalho remunerado.

mensuradas 20. Nas análises das estimativas padronizadas para a construção das variáveis latentes deste estudo (condição social ao longo da vida e condição de saúde ao longo da vida) considerou-se a carga fatorial e os autovalores. Todas as variáveis latentes resultaram de fatores com autovalores maiores do que 1,00 e todos os itens dos fatores apresentaram cargas fatoriais maiores do que 0,70.

O modelo de equação estrutural também permite a estimação de múltiplas funções lineares, incluindo efeitos diretos e indiretos, característica não permitida pelos métodos tradicionais de regressão. O método combina aspectos de regressão múltipla e análise fatorial para estimar as relações entre cada variável dependente (endógena) e as variáveis explicativas (exógenas). Para esta análise, as variáveis consideradas como endógenas foram condição de saúde ao longo da vida, esforço físico intenso no trabalho ao longo da vida, aposentadoria, capacidade para o trabalho, interação social primária, interação social secundária e trabalho remunerado.

Neste estudo, utilizou-se uma versão específica do modelo de equação estrutural para dados binários proposta por Kupek 15. Realizou-se inicialmente regressões logísticas com todas as variáveis do modelo conceitual. Num segundo momento, as variáveis com valor de $\mathrm{p}>0,05$ foram excluídas do modelo. Logo após essa etapa, realizou-se novamente as estimações das regressões logísticas do modelo somente com as variáveis que foram significativas. Na terceira etapa, os coeficientes do modelo de equação estrutural foram calculados com base nas odds ratio (OR) encontradas no segundo passo. Na quarta etapa, os coeficientes menores ou iguais a 0,18 foram excluídos do modelo. Após o desenvolvimento do modelo final foram calculados os coeficientes das associações indiretas e total, denominados coeficientes padronizados. Esse cálculo foi realizado baseando-se na transformação de 
Yule. Esse método aproxima a matriz dos coeficientes de correlação de Pearson das OR pela fórmula $(\mathrm{OR}-1) /(\mathrm{OR}+1) 15$. Quanto maior o valor do coeficiente padronizado, mais forte é a associação entre a variável explicativa e a variável dependente.

Para determinar se o modelo apresentou bom ajuste foram considerados os seguintes critérios: (a) a qualidade de ajuste de cada equação de regressão logística, com base nos valores de p, nos R2 e na comparação dos qui-quadrados dos modelos; (b) foram incluídas nos modelos de equação estrutural finais apenas as variáveis com coeficientes maiores ou iguais a 0,18. Decidiu-se assumir uma postura mais conservadora para que o modelo fosse mais parcimonioso. A busca da parcimônia nesse modelo se justifica pelo fato de não ser possível o cálculo de medidas gerais de ajuste, pois o modelo utilizado proposto por Kupek 15 baseia-se na transformação de estimadores de regressão logística (especificamente, as razões de chance) em um modelo de equações estruturais. Assim, só é possível ter indicadores de ajuste para cada equação isoladamente.

Todas as análises foram realizadas separadamente para homens e mulheres. As estimativas foram realizadas usando-se os procedimentos para amostras complexas do programa Stata 14.1 (https:// www.stata.com), de forma a considerar o efeito do delineamento do estudo e os pesos dos indivíduos.

\section{Resultados}

A amostra final deste estudo foi composta por 8.903 participantes do inquérito de base do ELSI-Brasil, ou seja, aquelas pessoas que possuíam informações completas para todas as variáveis. Desses, 4.961 (56\%) eram mulheres e 3.942 (44\%) eram homens. O trabalho remunerado foi informado por 31,3\% dos participantes, sendo maior para o sexo masculino $(39,6 \%$ em relação a $24,2 \%$ para mulheres; p < 0,001). Na Tabela 1, são mostradas as características dos participantes do estudo, segundo o gênero. Foram observadas diferenças estatisticamente significantes entre homens e mulheres para residência urbana aos 10 anos, escolaridade do indivíduo, idade em que começou a trabalhar, idade na data da entrevista, posição no domicílio, situação conjugal, condição de saúde ao longo da vida, número de doenças crônicas, esforço físico intenso no trabalho ao longo da vida e interação social primária.

Entre os homens, o trabalho remunerado apresentou associação significativa com condição social ao longo da vida, condição de saúde ao longo da vida, capacidade para o trabalho e interação social secundária. A situação conjugal e a idade na data da entrevista compuseram o modelo final como variáveis de ajuste. Para as mulheres, o trabalho remunerado apresentou associação significativa com condição social ao longo da vida, condição de saúde ao longo da vida, interação social primária, esforço físico intenso no trabalho ao longo da vida, capacidade para o trabalho e aposentadoria. A idade na data da entrevista compôs o modelo final como variável de ajuste. Os modelos conceituais finais das inter-relações entre os fatores associados à participação no trabalho remunerado, segundo o gênero, estão representados na Figura 2.

Na Tabela 2, são apresentadas as OR (IC95\%) e os coeficientes padronizados (CP) do modelo de equação estrutural das variáveis que compuseram o modelo final dos homens e das mulheres.

$\mathrm{Na}$ Tabela 3, estão apresentadas as associações diretas, indiretas e totais das variáveis associadas ao trabalho remunerado para homens e mulheres. De modo geral, os resultados mostraram maior associação entre a participação no trabalho remunerado das mulheres com a condição social ao longo da vida, apresentando um coeficiente padronizado (CP) total de 0,489, e a participação dos homens registrou maior associação com a capacidade para o trabalho $(\mathrm{CP}=0,527)$. Entre os homens, a condição social ao longo da vida apresentou associação indireta e positiva $(\mathrm{CP}=0,352)$ com o trabalho remunerado, principalmente via capacidade para o trabalho $(\mathrm{CP}=0,186)$. Para esse grupo, a condição social ao longo da vida também registrou associação indireta e positiva com o trabalho remunerado pela via da condição de saúde ao longo da vida e capacidade para o trabalho $(\mathrm{CP}=0,111)$. Entre as mulheres, a condição social ao longo da vida apresentou associação total $(\mathrm{CP}=0,489)$ e direta $(\mathrm{CP}=$ $0,257)$ positivas com o trabalho remunerado. Além disso, apresentou associação indireta e positiva $(\mathrm{CP}=0,232)$, principalmente via esforço físico intenso no trabalho ao longo da vida e aposentadoria $(\mathrm{CP}=0,076)$.

Entre homens e mulheres a condição de saúde ao longo da vida registrou associação indireta e positiva $(C P=0,298$ entre homens; $C P=0,142$ entre mulheres) com o trabalho remunerado, via 
Tabela 1

Distribuição do trabalho remunerado e demais características da população com 50 anos ou mais, segundo o gênero. Estudo Longitudinal da Saúde dos Idosos Brasileiros (ELSI-Brasil), 2015-2016.

\begin{tabular}{|c|c|c|c|c|c|c|c|}
\hline \multirow[t]{2}{*}{ Variáveis } & \multicolumn{2}{|c|}{ Total } & \multicolumn{2}{|c|}{ Homens } & \multicolumn{2}{|c|}{ Mulheres } & \multirow{2}{*}{$\begin{array}{c}\text { Valor de } \\
\text { p** }\end{array}$} \\
\hline & $\%$ * & IC95\% & $\%$ * & IC95\% & $\%$ * & IC95\% & \\
\hline Trabalho remunerado & 31,3 & $28,2-34,5$ & 39,6 & $36,0-43,4$ & 24,2 & $21,7-26,8$ & $<0,001$ \\
\hline Melhor condição social ao longo da vida & 41,1 & $38,1-44,2$ & 41,6 & $38,1-45,3$ & 40,6 & $37,5-43,9$ & 0,516 \\
\hline Anos de escolaridade da mãe (5+ anos) & 7,1 & $6,1-8,4$ & 7,9 & $6,4-9,7$ & 6,5 & $5,4-7,8$ & 0,117 \\
\hline Residência urbana aos 10 anos & 39,7 & $36,6-42,8$ & 38,1 & $34,9-41,4$ & 41,0 & $37,5-44,6$ & 0,043 \\
\hline Anos de escolaridade do participante (8+ anos) & 35,7 & $32,9-38,7$ & 37,4 & $34,0-40,9$ & 34,3 & $31,4-37,3$ & 0,033 \\
\hline Idade em que começou a trabalhar (18+ anos) & 17,5 & $15,4-19,8$ & 12,1 & $10,5-13,8$ & 22,3 & $19,7-25,2$ & $<0,001$ \\
\hline Idade na data da entrevista (65+ anos) & 35,9 & $32,2-39,7$ & 33,3 & $28,7-38,3$ & 38,1 & $35,0-41,3$ & 0,006 \\
\hline Responsável pelo domicílio & 73,2 & $71,4-75,0$ & 86,7 & $84,5-88,7$ & 61,7 & $57,9-65,4$ & $<0,001$ \\
\hline Vive com cônjuge/companheiro(a) & 63,5 & $60,5-66,3$ & 75,2 & $72,5-77,7$ & 53,5 & $50,4-56,5$ & $<0,001$ \\
\hline Residência atual urbana & 84,7 & $79,4-88,8$ & 84,8 & $79,2-89,1$ & 84,6 & $79,2-88,8$ & 0,855 \\
\hline Melhor condição de saúde ao longo da vida & 49,3 & $46,9-51,8$ & 52,6 & $49,6-55,4$ & 46,6 & $43,9-49,3$ & $<0,001$ \\
\hline Saúde boa até os 15 anos & 78,0 & $76,4-79,6$ & 78,8 & $76,9-80,7$ & 77,3 & $75,4-79,1$ & 0,139 \\
\hline Nenhuma ou uma doença crônica *** & 39,1 & $36,9-41,3$ & 49,7 & $47,2-52,1$ & 30,1 & $28,0-32,3$ & $<0,001$ \\
\hline Autoavaliação da saúde atual como boa & 43,7 & $41,3-46,2$ & 43,6 & $40,3-46,9$ & 43,8 & $41,4-46,3$ & 0,873 \\
\hline Esforço físico intenso no trabalho ao longo da vida & 19,3 & $17,1-21,8$ & 27,8 & $24,6-31,2$ & 11,9 & $9,8-14,4$ & $<0,001$ \\
\hline Capacidade para o trabalho boa & 48,4 & $46,0-50,8$ & 49,0 & $45,9-52,0$ & 47,8 & $45,5-50,2$ & 0,381 \\
\hline Aposentadoria & 54,4 & $51,3-57,5$ & 54,3 & $50,4-58,1$ & 54,6 & $51,0-58,1$ & 0,877 \\
\hline Interação social primária elevada & 48,4 & $46,4-50,5$ & 44,6 & $42,2-47,1$ & 51,6 & $49,2-54,1$ & $<0,001$ \\
\hline Interação social secundária elevada & 50,3 & $47,2-53,5$ & 49,0 & $45,2-52,8$ & 51,5 & $48,3-54,6$ & 0,087 \\
\hline Total & \multicolumn{2}{|c|}{8.903} & \multicolumn{2}{|c|}{3.942} & \multicolumn{2}{|c|}{4.961} & \\
\hline
\end{tabular}

IC95\%: intervalo de 95\% de confiança.

* Porcentagens ponderadas pelos parâmetros amostrais;

** Teste qui-quadrado de Pearson ou teste t de student com nível de significância $\leq$ 0,05; para diferenças entre gêneros;

*** Diagnóstico médico de hipertensão, diabetes, colesterol, acidente vascular cerebral, asma, artrite ou reumatismo, osteoporose, problema crônico de coluna, câncer, doença do coração e depressão.

capacidade para o trabalho. Ressalta-se que a força da associação para o grupo dos homens foi o dobro da magnitude da associação do grupo das mulheres. Em relação à interação social, a participação masculina no trabalho remunerado esteve associada direta e positivamente à interação social secundária $(\mathrm{CP}=0,209)$, já a participação feminina mostrou associação indireta e positiva com a interação social primária $(\mathrm{CP}=0,047)$. Apenas para o grupo das mulheres, aposentadoria apresentou associação direta e negativa $(\mathrm{CP}=-0,439)$ com o trabalho remunerado. Além disso, o trabalho remunerado também esteve associado indireta e negativamente com esforço físico intenso no trabalho ao longo da vida, via aposentadoria $(\mathrm{CP}=-0,156)$. 


\section{Figura 2}

Resultado do modelo de equação estrutural final com coeficientes padronizados dos fatores associados à participação no trabalho remunerado, segundo o gênero. Estudo Longitudinal da Saúde dos Idosos Brasileiros (ELSI-Brasil), 2015-2016.

2a) Homens

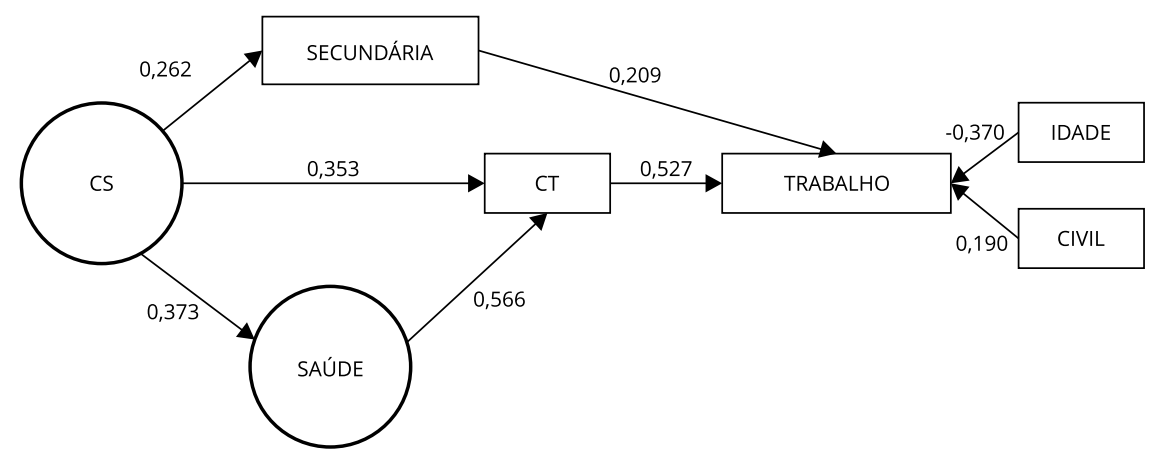

2b) Mulheres

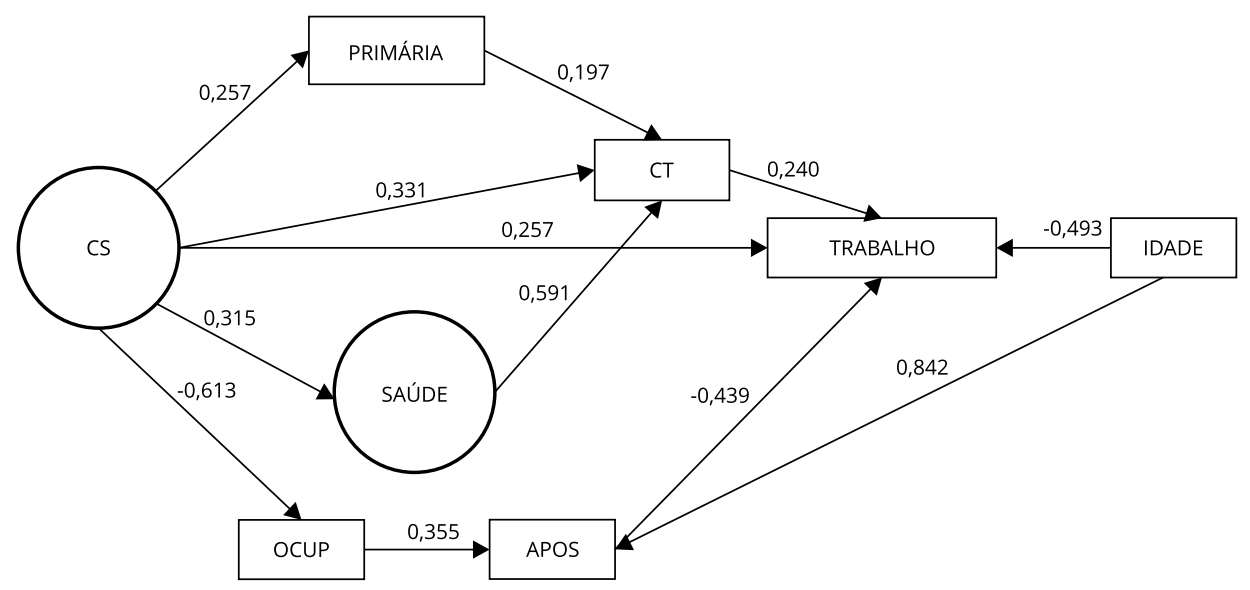

Fonte: elaboração própria dos autores (2018).

APOS: aposentadoria; CIVIL: situação conjugal; CS: condição social ao longo da vida; CT: capacidade para o trabalho; IDADE: idade na data da entrevista; OCUP: esforço físico intenso no trabalho ao longo da vida; SAÚDE: condição de saúde ao longo da vida; PRIMÁRIA: interação social primária; SECUNDÁRIA: interação social secundária; TRABALHO: trabalho remunerado.

Nota: $\mathrm{N}=8.903$ (3.942 homens e 4.961 mulheres). 
Tabela 2

Resultados da regressão logística multivariada e respectivo coeficiente de padronização do modelo de equação estrutural para cada variável endógena, segundo o gênero. Estudo Longitudinal da Saúde dos Idosos Brasileiros (ELSI-Brasil), 2015-2016.

\begin{tabular}{|c|c|c|c|c|c|c|c|}
\hline \multirow[t]{2}{*}{ Variáveis endógenas * } & \multirow[t]{2}{*}{ Variáveis exógenas ** } & \multicolumn{3}{|c|}{ Homens } & \multicolumn{3}{|c|}{ Mulheres } \\
\hline & & OR *** & IC95\% & CP \# & OR *** & IC95\% & CP \# \\
\hline $\begin{array}{l}\text { Condição de saúde ao } \\
\text { longo da vida }\end{array}$ & Condição social ao longo da vida & 2,19 & $1,86-2,58$ & 0,37 & 1,92 & $1,65-2,24$ & 0,32 \\
\hline $\begin{array}{l}\text { Esforço físico intenso no } \\
\text { trabalho ao longo da vida }\end{array}$ & Condição social ao longo da vida & - & - & - & 0,24 & $0,17-0,33$ & $-0,61$ \\
\hline \multirow[t]{2}{*}{ Aposentadoria } & $\begin{array}{l}\text { Esforço físico intenso no trabalho } \\
\text { ao longo da vida }\end{array}$ & - & - & - & 2,09 & $1,70-2,58$ & 0,36 \\
\hline & Idade na data da entrevista & - & - & - & 11,69 & $\begin{array}{l}9,59- \\
14,25\end{array}$ & 0,84 \\
\hline \multirow[t]{3}{*}{ Capacidade para o trabalho } & Condição social ao longo da vida & 2,09 & $1,82-2,40$ & 0,35 & 1,99 & $1,66-2,38$ & 0,33 \\
\hline & $\begin{array}{l}\text { Condição de saúde ao longo da } \\
\text { vida }\end{array}$ & 3,61 & $3,06-4,26$ & 0,57 & 3,89 & $3,23-4,56$ & 0,59 \\
\hline & Interação social primária & - & - & - & 1,49 & $1,26-1,77$ & 0,20 \\
\hline Interação social primária & Condição social ao longo da vida & - & - & - & 1,69 & $1,41-2,02$ & 0,26 \\
\hline Interação social secundária & Condição social ao longo da vida & 1,71 & $1,42-2,05$ & 0,26 & - & & - \\
\hline \multirow[t]{6}{*}{ Trabalho remunerado } & Condição social ao longo da vida & - & - & - & 1,69 & $1,40-2,03$ & 0,26 \\
\hline & Aposentadoria & - & - & - & 0,39 & $0,32-0,47$ & $-0,44$ \\
\hline & Capacidade para o trabalho & 3,23 & $2,71-3,86$ & 0,53 & 1,63 & $1,37-1,95$ & 0,24 \\
\hline & Interação social secundária & 1,53 & $1,30-1,81$ & 0,21 & - & - & - \\
\hline & Vive com cônjuge/companheiro(a) & 1,47 & $1,17-1,86$ & 0,19 & - & - & - \\
\hline & Idade na data da entrevista & 0,46 & $0,34-0,61$ & $-0,37$ & 0,34 & $0,27-0,42$ & $-0,49$ \\
\hline
\end{tabular}

CP: coeficiente padronizado; IC95\%: intervalo de 95\% de confiança; OR: odds ratio.

Nota: $\mathrm{N}=8.903$ (3.942 homens e 4.961 mulheres).

* Variáveis endógenas: variáveis dependentes para cada regressão logística;

** Variáveis exógenas: variáveis explicativas para cada regressão logística;

*** OR ajustada por todas as variáveis listadas como explicativas pela regressão logística, com variância robusta;

$\# C P=(O R-1) /(O R+1)$.

\section{Discussão}

Os resultados deste estudo mostraram que a participação no trabalho remunerado de homens e mulheres com 50 anos ou mais ocorre por mecanismos diferentes. A participação feminina foi influenciada diretamente pela condição social ao longo da vida, capacidade para o trabalho e aposentadoria, e indiretamente pela condição de saúde ao longo da vida, esforço físico intenso no trabalho ao longo da vida e interação social primária. Por outro lado, a participação masculina no trabalho remunerado foi influenciada diretamente pela capacidade para o trabalho e interação social secundária, e indiretamente pela condição social ao longo da vida e condição de saúde ao longo da vida. Segundo os resultados do modelo de equação estrutural, a variável que apresentou maior força de associação com a participação feminina no trabalho remunerado foi a condição social ao longo da vida, já que para os homens foi a capacidade para o trabalho. Para ambos os gêneros, a condição de saúde ao longo da vida apresentou associação indireta e positiva com o trabalho remunerado, mas a magnitude da força da associação para os homens foi duas vezes maior que para as mulheres. Os resultados do modelo de equação estrutural identificaram ainda diferenças de gênero em relação à interação social, com a participação dos homens no trabalho remunerado caracterizada pelo engajamento em atividades sociais organizadas ou associações civis, e a participação das mulheres caracterizada pela relação com parentes e amigos próximos. Além disso, somente entre as mulheres observou-se associação negativa 
Tabela 3

Resultados do modelo de equação estrutural com as associações diretas, indiretas e totais das variáveis associadas ao trabalho remunerado, segundo o gênero. Estudo Longitudinal da Saúde dos Idosos Brasileiros (ELSI-Brasil), 2015-2016.

\begin{tabular}{|c|c|c|c|c|c|c|}
\hline \multirow[t]{2}{*}{ Associação das variáveis } & \multicolumn{3}{|c|}{ Homens } & \multicolumn{3}{|c|}{ Mulheres } \\
\hline & $\begin{array}{c}\text { CP } \\
\text { associação } \\
\text { direta }\end{array}$ & $\begin{array}{c}\text { CP } \\
\text { Associação } \\
\text { indireta }\end{array}$ & $\begin{array}{c}\text { CP } \\
\text { Associação } \\
\text { total }\end{array}$ & $\begin{array}{c}\text { CP } \\
\text { Associação } \\
\text { direta }\end{array}$ & $\begin{array}{c}\text { CP } \\
\text { Associação } \\
\text { indireta }\end{array}$ & $\begin{array}{c}\text { CP } \\
\text { Associação } \\
\text { total * }\end{array}$ \\
\hline $\mathrm{CS} \rightarrow \mathrm{TR}$ & - & - & 0,352 & 0,257 & 0,232 & 0,489 \\
\hline $\mathrm{CS} \rightarrow \mathrm{CT} \rightarrow \mathrm{TR}$ & - & 0,186 & - & - & 0,079 & - \\
\hline $\mathrm{CS} \rightarrow \mathrm{OCUP} \rightarrow \mathrm{APOS} \rightarrow \mathrm{TR}$ & - & - & - & - & 0,076 & - \\
\hline $\mathrm{CS} \rightarrow \mathrm{SAÚDE} \rightarrow \mathrm{CT} \rightarrow \mathrm{TR}$ & - & 0,111 & - & - & 0,045 & - \\
\hline $\mathrm{CS} \rightarrow$ PRIMÁRIA $\rightarrow \mathrm{CT} \rightarrow \mathrm{TR}$ & - & - & - & - & 0,012 & - \\
\hline $\mathrm{CS} \rightarrow \mathrm{SECUNDÁRIA} \rightarrow$ TR & - & 0,055 & - & - & - & - \\
\hline $\mathrm{SAÚDE} \rightarrow \mathrm{CT} \rightarrow \mathrm{TR}$ & - & 0,298 & - & - & 0,142 & - \\
\hline $\mathrm{CT} \rightarrow \mathrm{TR}$ & 0,527 & - & - & 0,249 & - & - \\
\hline $\mathrm{APOS} \rightarrow \mathrm{TR}$ & - & - & - & $-0,439$ & - & - \\
\hline $\mathrm{OCUP} \rightarrow \mathrm{APOS} \rightarrow \mathrm{TR}$ & - & - & - & - & $-0,156$ & - \\
\hline PRIMÁRIA $\rightarrow \mathrm{CT} \rightarrow \mathrm{TR}$ & - & - & - & - & 0,047 & - \\
\hline SECUNDÁRIA $\rightarrow$ TR & 0,209 & - & - & - & - & - \\
\hline
\end{tabular}

APOS: aposentadoria; CP: coeficiente padronizado **; CS: condição social ao longo da vida; CT: capacidade para o trabalho;

OCUP: esforço físico intenso no trabalho ao longo da vida; PRIMÁRIA: interação social primária;

SAÚDE: condição de saúde ao longo da vida; SECUNDÁRIA: interação social secundária; TR: trabalho remunerado.

Nota: $\mathrm{N}=8.903$ (3.942 homens e 4.961 mulheres).

* Associação total = associação direta + associação indireta;

** $C P=(O R-1) /(O R+1)$

entre fatores relacionados ao trabalho (esforço físico intenso no trabalho ao longo da vida e aposentadoria) e participação no trabalho remunerado.

A condição social ao longo da vida parece afetar a participação no trabalho remunerado de homens e mulheres em idades avançadas 1. Uma pesquisa conduzida na Região Metropolitana de Belo Horizonte 21 mostrou que para homens e mulheres, tanto o rendimento, quanto o nível de escolaridade dos pais garante a educação dos filhos. No entanto, a participação das mulheres no trabalho remunerado, em qualquer idade, depende mais do seu nível socioeconômico, em particular da escolaridade, em comparação ao nível socioeconômico dos pais (renda e escolaridade). O presente estudo mostrou que a condição social ao longo da vida apresentou associação direta com o trabalho remunerado apenas entre as mulheres, além de registrar associação indireta via esforço físico intenso no trabalho ao longo da vida e aposentadoria. Esses resultados indicam a importância da condição social ao longo da vida para a participação feminina no trabalho remunerado. Independentemente de outros fatores, as mulheres com melhor condição social ao longo da vida apresentam maior probabilidade de participar do trabalho remunerado em idades avançadas. Além disso, os resultados evidenciam que mulheres com melhor condição social ao longo da vida apresentam menor probabilidade de exercer esforço físico intenso no trabalho ao longo da vida, levando à menor probabilidade de aposentadoria e, consequentemente, maior probabilidade de participar do trabalho remunerado. Em relação aos homens a condição social ao longo da vida apresentou somente associação indireta, via condição de saúde ao longo da vida e capacidade para o trabalho. Os resultados sugerem que homens com melhor condição social ao longo da vida registram melhor condição de saúde ao longo da vida, percepção da capacidade para o trabalho como boa e maior probabilidade de participar do trabalho remunerado.

A saúde é considerada o principal fator determinante da capacidade para o trabalho 4,22 , e um nível de saúde ruim aumenta a probabilidade de deterioração desta capacidade 23 . Estudos mostram que independentemente de como a saúde é medida, seja objetiva ou subjetivamente 24 , ela continua sendo um fator determinante da participação no trabalho remunerado em idades avançadas 25 . 
Os resultados do presente estudo sugerem que, tanto homens quanto mulheres com melhores condições de saúde ao longo da vida tendem a perceber sua capacidade para o trabalho como boa, apresentando maior probabilidade de participar do trabalho remunerado. Os resultados do modelo de equação estrutural mostraram ainda que, para os homens, a força da associação entre condição de saúde ao longo da vida e participação no trabalho remunerado foi o dobro da magnitude da associação para as mulheres. O que pode ser explicado pelo fato de que os homens tendem a exercer um trabalho mais desgastante fisicamente ao longo da vida, quando comparados às mulheres.

Segundo um estudo baseado na teoria do capital social 26 , o fato de um indivíduo pertencer a grupos ou associações (interação social secundária) afeta positivamente a probabilidade de ter uma maior participação no trabalho remunerado. Relacionamentos baseados em laços fracos (secundários), como aqueles encontrados entre os participantes de um grupo ou associação, são importantes pois conectam as pessoas a uma maior gama de informações e conhecimentos relativos a emprego. Apesar dos amigos próximos de um indivíduo serem mais motivados a ajudá-lo, os laços fracos estão estruturalmente localizados de tal modo a serem mais úteis no processo de busca por emprego 26 . O presente estudo acrescentou a esses achados, uma vez que mostrou que a participação dos homens no trabalho remunerado está direta e positivamente associada à interação social secundária. Ao passo que, entre as mulheres, ter contato mais frequente com família e amigos (interação social primária) aumenta a probabilidade da participação feminina no trabalho remunerado, via capacidade para o trabalho 26. Parece que, para as mulheres, o contexto social formado pela família e amigos pode ser importante para a percepção da sua capacidade para o trabalho e consequente participação no trabalho remunerado. Isso, provavelmente, deve-se ao fato de que no modelo de família patriarcal, que é o predominante, o funcionamento da família é uma atribuição feminina. Uma interação mais forte das mulheres dentro de redes primárias pode, assim, garantir às mulheres de maior idade a segurança de que a funcionalidade familiar pode acontecer mesmo com sua ausência mais prolongada do ambiente doméstico. Nesse caso, não se pode descartar a possibilidade de causalidade reversa, uma vez que a interação social pode ser causa ou consequência da maior participação no trabalho remunerado.

Somente entre as mulheres, a associação entre condição social ao longo da vida e trabalho remunerado foi parcialmente mediada pelo esforço físico intenso no trabalho ao longo da vida e pela aposentadoria. Mulheres que exerceram esforço físico intenso no trabalho ao longo da vida apresentaram maior probabilidade de estar aposentada e, consequentemente, menor probabilidade de participar do trabalho remunerado. Há evidências de que os fatores psicossociais e físicos do trabalho, como alta demanda física e baixo controle, podem mediar a associação entre posição social de um indivíduo e o risco de aposentadoria por invalidez 2 . Os resultados deste estudo reforçam a hipótese de que o trabalho remunerado pode representar uma sobrecarga para a saúde das mulheres quando a qualidade do emprego é baixa, e estar associada ao trabalho doméstico para a própria família 2,27.

A principal vantagem deste estudo é a sua grande base populacional, representativa da população brasileira com 50 anos ou mais. Ao nosso conhecimento, este é o primeiro estudo baseado em amostra nacional a analisar as relações entre os determinantes da participação no trabalho remunerado entre adultos mais velhos. Outra vantagem do estudo é o uso do modelo de equações estruturais, que permite analisar, simultaneamente, os fatores associados ao trabalho remunerado. Entre as limitações do estudo podemos citar o viés de informação e o viés de desejabilidade social, isto é, o desejo de transmitir uma imagem social e culturalmente aceitável. Outra limitação diz respeito à sua natureza transversal, que não permite estabelecer associações temporais para as variáveis relativas às condições atuais. Finalmente, uma terceira limitação do estudo é que a especificação do modelo final analisado não contou com uma medida geral de ajuste.

Como recomendações finais, os resultados do modelo de equação estrutural mostraram a complexidade da participação no trabalho remunerado de pessoas com 50 anos ou mais, principalmente em relação às diferenças de gênero. Para o grupo das mulheres, investimentos na condição social ao longo da vida, em particular na escolaridade, podem contribuir para aumentar a participação nas atividades econômicas. Além disso, estudos sobre a participação feminina no mercado de trabalho devem considerar o tipo de trabalho ao longo da vida e entender melhor o papel da aposentadoria na vida das mulheres. Por outro lado, a participação dos homens mais velhos no mercado de trabalho depende mais de melhorias nas condições de saúde ao longo da vida e na capacidade para o trabalho. Pesquisas que analisam a participação de adultos mais velhos no mercado de trabalho devem abordar 
uma perspectiva do curso de vida, considerando os fatores nos níveis micro, meso e macro. Além disso, devem verificar se as relações entre os fatores associados a essa participação se mantêm ao longo do tempo.

Concluindo, apesar de diferenças importantes nos determinantes do trabalho remunerado entre homens e mulheres mais velhos, as condições de saúde ao longo da vida apresentaram associações indiretas e positivas em ambos os gêneros, via capacidade para o trabalho. Esses resultados sugerem que melhorias nas condições de saúde em todas as idades têm o potencial de aumentar a participação de adultos mais velhos brasileiros no mercado de trabalho.

\section{Colaboradores}

Todos os autores contribuíram igualmente para todas as fases do estudo e na elaboração e revisão do manuscrito.

\section{Informações adicionais}

ORCID: Camila Menezes Sabino de Castro (00000002-5383-9796); Maria Fernanda Lima-Costa (0000-0002-3474-2980); Jorge Alexandre Barbosa Neves (0000-0003-4141-2282); Fabíola Bof de Andrade (0000-0002-3467-3989); Rosana Ferreira Sampaio (0000-0002-4775-9650).

\section{Agradecimentos}

M. F. F. L. Castro, J. A. B. Neves e R. F. Sampaio são bolsistas de produtividade em pesquisa do Conselho Nacional de Desenvolvimento Científico e Tecnológico, Ministério da Saúde (DECIT/SCTIE); COSAPI/DAPES/SAS; Ministério da Ciência, Tecnologia, Inovação e Comunicação.

\section{Referências}

1. Camarano AA, Kanso S, Fernandes D. Menos jovens e mais idosos no mercado de trabalho. In: Camarano AA, organizador. Novo regime demográfico: uma nova relação entre população e desenvolvimento? Rio de Janeiro: Instituto de Pesquisa Econômica Aplicada; 2014. p. 377-406.

2. Hasselhorn HM, Apt W. Understanding employment participation of older workers: creating a knowledge base for future labour market challenges. http://www.jp-demographic. eu/ (acessado em 10/Mai/2018).

3. Nonato F, Pereira RHM, Nascimento PAMM, Araújo TC. O perfil da força de trabalho brasileira: trajetórias e perspectivas. Boletim de mercado de trabalho - conjuntura e análise. Rio de Janeiro: Instituto de Pesquisa Econômica Aplicada; 2012.

4. Ilmarinem JE. Aging workers. https://oem. bmj.com/content/58/8/546 (acessado em 29/ $\mathrm{Jul} / 2019)$.

5. Department of Economic and Social Affairs, United Nations. World population ageing 2017. http://esa.un.org/wpp (acessado em 10/ Mai/2018).

6. Instituto Brasileiro de Geografia e Estatística. Pesquisa Nacional por Amostra de Domicílios Contínua. 3o trimestre de 2019. Rio de Janeiro: Instituto Brasileiro de Geografia e Estatística; 2019.

7. Bélanger A, Carrière Y, Sabourin P. Understanding employment participation o folder workers: the Canadian perspective. Can Public Policy 2016; 42:94-109. 
8. Ybema, JF, Geuskens GA, van den Heuvel S, Wind A, Leijten FRM, et al. Study on Transitions in Employment, Ability and Motivation (STREAM): the design of a four-year longitudinal cohort study among 15.118 persons aged 45 to 64 years. British Journal of Medicine \& Medical Research 2014; 4:1383-99.

9. Ploubidis GB, Silverwood RJ, DeStavola B, Grundy E. Life-course partnership status and biomarkers in midlife: evidence from the 1958 British Birth Cohort. Am J Public Health 2015; 105:1596-603.

10. World Health Organization. Active ageing: a policy framework, 2002. http://www.who. int/ageing/publications/en (acessado em 26/ Abr/2018).

11. Bem-Shlomo Y, Kuh D. A life course approach to chronic disease epidemiology: conceptual models, empirical challenges and interdisciplinar perspectives. Int J Epidemiol 2002; 31:285-93.

12. Pérez ER, Wajnman S, Oliveira AMHC. Análise dos determinantes da participação no mercado de trabalho dos idosos em São Paulo. Rev Bras Estud Popul 2006; 23:269-86.

13. Castro CMS, Mambrini JVM, Sampaio RF, Macinko J, Lima-Costa MF. Aspectos sociodemográficos e de saúde associados ao trabalho remunerado em adultos (50-69 anos) na Região Metropolitana de Belo Horizonte, Minas Gerais, Brasil. Cad Saúde Pública 2015; 31:1775-87.

14. Wind A, Geuskens GA, Ybema JF, Bongers PM, van der Beek AJ. The role of ability, motivation, and opportunity to work in the transition from work to early retirement - testing and optimizing the Early Retirement Model. Scand J Work Environ Health 2015; 41:24-35.

15. Kupek E. Beyond logistic regression: structural equations modelling for binary variables and its application to investigating unobserved confounders. BMC Med Res Methodol 2006; 6:13.

16. Amorim LDAF, Fiaccone RL, Santos CAST, Santos TN, Moraes LTLP, Oliveira NF, et al. Structural equation modeling in epidemiology. Cad Saúde Pública 2010; 26:2251-62.

17. Morais AODS, Simões VMF, Rodrigues LS, Batista RFL, Lamy ZC, Carvalho CA, et al. Sintomas depressivos e de ansiedade maternos e prejuízos na relação mãe/filho em uma coorte pré-natal: uma abordagem com modelagem de equações estruturais. Cad Saúde Pública 2017; 33:e00032016.
18. Lima-Costa MF, Andrade FB, Souza Jr. PRB, Neri AL, Duarte YAO, Castro-Costa E, et al. The Brazilian Longitudinal Study of Aging (ELSI-Brazil): objectives and design. Am J Epidemiol 2018; 187:1345-53.

19. Granovetter M. The strength of the weak ties. Am J Sociol 1973; 78:1360-80.

20. Silva AAM, Vasconcelos AGG, Bettiol H, Barbieri MA. Socioeconomic status, birth weight, maternal smoking during pregnancy and adiposity in early adult life: an analysis using structural equation modeling. Cad Saúde Pública 2010; 26:15-29.

21. Aguiar NF, Fernandes DC, Neves JAB. Mobilidade social feminina. In: Aguiar NF, organizador. Desigualdades sociais, redes de sociabilidade e participação política. v. 1. Belo Horizonte: Editora da UFMG; 2007. p. 165-80.

22. Martinez MC, Latorre MRDO. Saúde e capacidade para o trabalho em trabalhadores de área administrativa. Rev Saúde Pública 2006; 40:851-8.

23. Gould R, Ilmarinen J, Järvisalo J, Koskinen S, editors. Dimension of work ability: results of the health 2000 survey. Helsinki: Finnish Centre of Pensions/The Social Insurance Institution/National Public Health Institute/Finnish Institute of Occupational Health; 2008.

24. Lima-Costa MF, Cesar CC, Chor D, Proietti FA. Self-rated health compared with objectively measured health status as a tool for mortality risk screening in older adults: 10-year follow-up of the Bambuí Cohort Study of Aging. Am J Epidemiol 2012; 175:228-35.

25. Castro CMS, Lima-Costa MF, César CC, Neves JAB, Bof de Andrade F, Souza-Junior PRB, et al. Curso da vida e capacidade para o trabalho entre adultos mais velhos: ELSI-Brasil. Rev Saúde Pública 2018; 52 Suppl 2:11s.

26. Neves JAB, Helal DH. Associativismo, capital social e mercado de trabalho. In: Aguiar NF, organizador. Desigualdades sociais, redes de sociabilidade e participação política. v. 1. Belo Horizonte: Editora da UFMG; 2007. p. 61-72.

27. Barata RB. Relações de gênero e saúde: desigualdade ou discriminação? In: Barata RB. Como e por que as desigualdades sociais fazem mal à saúde. Rio de Janeiro: Editora Fiocruz; 2009. p. 73-94. 


\section{Abstract}

This study aimed to develop a conceptual model and to explore direct and indirect associations between paid work and life-course factors in a representative national sample of the Brazilian population 50 years and older. The analysis was based on 8,903 participants in the baseline of the Brazilian Longitudinal Study of Aging (ELSI-Brazil). The exposure variables were sociodemographic, health, work, and social interaction variables. Based on a structural equation model, paid work showed total association with lifetime social status in women (standardized coefficient $-S C=0.489$ ) and direct association with capacity for work in men $(S C=0.527)$. For women alone, an indirect and negative association was observed with lifetime intense physical effort at work, via retirement $(S C=-0.156)$. Men with paid work were more prone to social participation, measured by belonging to groups or associations $(S C=0.209)$. Among women, this participation was through interaction with family and friends $(S C=0.047)$, via capacity for work. For both men and women, lifetime health status showed an indirect and positive association $(S C=0.298$ men; $S C=0.142$ women $)$ with paid work, via capacity for work. All the abovementioned factors showed a significant association with paid work. The study's results showed that participation in the work market occurs by different mechanisms for men and women, principally considering the factors related to work and social interaction.

Aging; Work; Statiscal Models; Socieconimic Status; Social Interaction

\section{Resumen}

El objetivo de este estudio fue desarrollar un modelo conceptual y explorar asociaciones directas $e$ indirectas entre trabajo remunerado y factores que operan a lo largo de la vida, en una muestra nacional representativa de población brasileña con 50 años y más. El análisis se basó en 8.903 participantes procedentes del Estudio Brasileño Longitudinal del Envejecimiento (ELSI-Brasil). Los factores de exposición fueron: sociodemográficos, de salud, trabajo e interacción social. Utilizando un modelo de ecuaciones estructurales, se observó que el trabajo remunerado presentó una asociación total con la condición social a lo largo de la vida entre mujeres (coeficiente de estandarización$C P=0,489)$ y asociación directa con la capacidad para el trabajo entre hombres $(C P=0,527)$. Solamente para las mujeres, la asociación indirecta y negativa se observó con el esfuerzo físico intenso en el trabajo a lo largo de la vida, vía jubilación $(C P=-0,156)$. Hombres con trabajo remunerado fueron más propensos a la participación social, medida por la pertenencia a grupos o asociaciones $(C P=0,209)$. Entre mujeres, esta participación se produjo mediante interacciones con la familia $y$ amigos $(C P=0,047)$, vía capacidad para el trabajo. Tanto entre hombres como entre mujeres, la condición de salud a lo largo de la vida mostró una asociación indirecta y positiva $(C P=0,298$ hombres; $C P=0,142$ mujeres) con el trabajo remunerado, vía capacidad para el trabajo. Todos los factores mencionados presentaron una asociación significativa con el trabajo remunerado. Los resultados del estudio mostraron que la participación en el mercado de trabajo de hombres y mujeres mayores se produce por mecanismos diferentes, principalmente al considerar los factores relacionados con el trabajo e interacción social.

Envejecimiento; Trabajo; Modelos Estadísticos; Situación Socioeconómica; Interacción Social
Recebido em 04/Out/2019

Versão final reapresentada em 14/Fev/2020

Aprovado em 01/Abr/2020 